\title{
Self-Reported Low Vitality, Poor Mental Health, and Low Dietary Restraint Are Associated with Overperception of Physical Exertion
}

\author{
Paula C. Chandler-Laney, ${ }^{1}$ David W. Brock, ${ }^{2,3}$ Barbara A. Gower, ${ }^{1}$ Jessica A. Alvarez, ${ }^{1}$ \\ Nikki C. Bush, ${ }^{1}$ and Gary R. Hunter ${ }^{4}$ \\ ${ }^{1}$ Department of Nutrition Sciences, University of Alabama-Birmingham, 1675 University Boulevard, WEBB 413, Birmingham, \\ AL 35294, USA \\ ${ }^{2}$ Department of Medicine, University of Alabama-Birmingham, 1675 University Boulevard, WEBB 413, Birmingham, AL 35294, USA \\ ${ }^{3}$ Department of Exercise and Movement Science, University of Vermont, Burlington, VT05405, USA \\ ${ }^{4}$ Department of Human Studies, University of Alabama-Birmingham, 1675 University Boulevard, WEBB 413, Birmingham, \\ AL 35294, USA
}

Correspondence should be addressed to Paula C. Chandler-Laney, pchandle@uab.edu

Received 16 April 2010; Revised 24 August 2010; Accepted 3 September 2010

Academic Editor: Jack Adam Yanovski

Copyright (C) 2010 Paula C. Chandler-Laney et al. This is an open access article distributed under the Creative Commons Attribution License, which permits unrestricted use, distribution, and reproduction in any medium, provided the original work is properly cited.

\begin{abstract}
Objective. We investigated whether perceived exertion, in comparison to the physiological response to exercise, was associated with self-reported vitality, mental health, and physical function during daily activities, or weight control behaviors. Design. Weightreduced, formerly overweight women $(n=126$, aged 22-46 years), completed health and dietary control questionnaires, and underwent a treadmill-walking task while heart rate, ventilation, respiratory exchange ratio, and ratings of perceived exertion were recorded. Results. Overperception of exertion (perceived exertion physiological exertion) was inversely associated with vitality $(r=-0.190, P<.05)$, mental health $(r=-0.188, P<.05)$, and dietary control $(r$ values range -0.231 to $-0.317, P<.05)$. In linear regression modeling, vitality or mental health, and cognitive dietary restraint were independently associated with accuracy of perceived exertion, independent of age, ethnicity, and engagement in exercise during weight loss. Each model explained 7\%-8\% of the variance in accuracy of perceived exertion. Conclusion. Women with low vitality or poor mental health, and poor dietary control may overperceive exertion. Such overperception may be a barrier to engage in physical activity and thus increase susceptibility to weight gain.
\end{abstract}

\section{Introduction}

The obesity epidemic has been attributed in part to sedentary lifestyles. While advances in technology and changes in the environment have promoted sedentary lifestyles, public health efforts have attempted to counter these changes by encouraging physical activity among the general population. Despite these efforts, the proportion of adults in the USA who meet daily physical activity recommendations is estimated to be between just $5 \%$ and $28 \%[1,2]$.

It is possible that people with low levels of physical activity do not engage in exercise because they perceive it as being difficult. Indeed, in a study of sedentary women, those with low activity-related energy expenditure tended to have high ratings of perceived exertion during standardized activities of daily living [3]. Whether high perceived exertion reflects true physiological exertion has not been investigated thoroughly. Borg rating scales are frequently used to measure perceived exertion and the 15-point scale specifically, is believed to reflect heart rate during a physical activity task [4-6]. It is likely, however, that other physiological indices such as ventilation and respiratory exchange ratio (RER), impact ratings of perceived exertion (RPE). Our group recently developed an index of the accuracy of perceived 
exertion by comparing a composite measure of physiological exertion encompassing heart rate, ventilation, and RER, to individuals' RPE [7]. In this study, it was found that even among a relatively homogenous sedentary, premenopausal female population, some tended to overperceive exertion, while others underperceived exertion [7]. Furthermore, women who perceived exertion to be greater than that implied by their physiological response were more likely to regain weight after a weight loss intervention [7].

Despite the potential importance of accuracy in perceived exertion to regulate engagement in physical activity, very few studies have investigated factors that might influence the accuracy of perceived exertion. It is feasible that an underlying psychological or behavioral profile might be associated with the tendency to over- or underperceive exertion. Equivocal results have been shown in investigations of personality constructs and perceived exertion [8-11]. However, one study found that women with an external locus of control had higher RPE during a cycle task as compared to those with an internal locus of control [11]. These authors also reported that women with an external locus of control reported greater RPE relative to heart rate than that expected in the general population. Although these findings support the hypothesis that an external locus of control is associated with the overperception of exertion, little research has explored this possibility further, and none has shown whether weightrelated locus of control specifically is related to accuracy in the perception of exertion. Furthermore, to our knowledge, associations between accuracy of perceived exertion and perceived physical and mental health, and self-reported weight control behaviors, have not been investigated.

The main objective of this study was to examine whether self-reported vitality, mental health, physical function during activities of daily life, or self-reported weight control behaviors, were associated with the accuracy of perceived exertion; a construct which has previously been shown to be an independent predictor of weight regain. Specifically, we hypothesized that those women who overperceived exertion would have lower vitality, poorer physical function, and poorer mental health, and would report poor weight control behaviors such as a lack of dietary control and an external weight locus of control (i.e., blaming external factors for their weight). This was investigated in a group of premenopausal sedentary women who had previously lost weight via an investigator-managed intervention.

\section{Methods}

2.1. Subjects. This investigation was conducted in women who were enrolled in a weight loss and weight loss maintenance study. Overweight (BMI $27-30 \mathrm{~kg} / \mathrm{m}^{2}$ ), premenopausal (22-46 years) women underwent a weight loss intervention in which they were provided with a $3347.2 \mathrm{~kJ} /$ day diet with or without exercise training. Women were maintained on this program until they achieved a BMI of $<25 \mathrm{~kg} / \mathrm{m}^{2}$. All women were nonsmokers and experienced regular menses. We used all available data from those women who had completed the weight loss phase $(n=126)$.
2.2. Procedure. Immediately following weight loss, women underwent a 4-week supervised weight maintenance period during which they were weighed 3-5 times per week. For the last 2 weeks of this period, subjects were provided with a control diet $(20 \%-22 \% \mathrm{~kJ}$ from fat, $18 \%-22 \% \mathrm{~kJ}$ from protein, $58 \%-62 \% \mathrm{~kJ}$ from carbohydrate) from the General Clinical Research Center (GCRC) kitchen. At the end of this weight maintenance period, subjects completed questionnaires regarding their perceived health and weight control behaviors, and underwent exercise and body composition testing. The protocol was approved by the University of Alabama at Birmingham Institutional Review Board and was compliant with the Human Services Regulation for Protection of Human Research Subjects. Informed consent was provided by each participant.

\subsection{Measures}

2.3.1. Treadmill Tests. Study participants underwent both a submaximal and a maximal test on a calibrated treadmill following an overnight fast. For the submaximal test, participants maintained a $4.84 \mathrm{~km} / \mathrm{h}(3 \mathrm{mph})$ pace for 4 minutes, while measurements of heart rate (HR), oxygen consumption $\left(\dot{\mathrm{V}}_{2}\right)$, ventilation $\left(\dot{\mathrm{V}}_{\mathrm{E}}\right)$, respiratory exchange ratio (RER), and ratings of perceived exertion (RPE), were obtained. Heart rate was measured using a heart rate monitor (Polar Vantage XL, Polar Beat, Port Washington, New York). Open-circuit spirometry using a MAX-II metabolic testing system (PHYSIO-DYNE, Quogue, New York) measured $\dot{\mathrm{V}}_{\mathrm{E}}$, $\dot{\mathrm{V}} \mathrm{O}_{2}$, and $\dot{\mathrm{V}} \mathrm{CO}_{2}$. Steady state $\dot{\mathrm{V}} \mathrm{O}_{2}$ was derived from the average of the $3 \mathrm{rd}$ and 4 th minutes. The RPE was collected during the 4 th minute using the 15-point Borg rating scale $[4,5]$. The $4.84 \mathrm{~km} / \mathrm{h}$ pace was chosen as a common velocity at which steady state oxygen uptake can be achieved by sedentary women [12]. All participants had experienced at least two treadmill walks prior to testing.

The maximal test involved the use of the modified Bruce protocol and has previously been described in [7]. In brief, participants began walking at $4.84 \mathrm{~km} / \mathrm{h}$ on the flat for 2 minutes, after which the grade was increased $2.5 \%$ each minute until voluntary exhaustion. Maximum oxygen uptake $\left(\dot{\mathrm{VO}}_{2} \max \right)$ was determined by indirect calorimetry (MAX-II CART). All participants reached at least 2 criteria for achieving $\mathrm{V}_{2}$ max, (plateauing of $\dot{\mathrm{V}}_{2}$, RER above 1.2, and heart rate within 10 beats of age predicted maximum).

RPE and the physiological exertion measures of HR, RER, and $\dot{V}_{\mathrm{E}}$ were obtained in the same manner as those used during the submaximal treadmill test.

2.3.2. Physiological Exertion. A composite score of physiological exertion was calculated in the manner described by Brock et al. [7]. In brief, we first calculated relative physiological effort during the submaximal treadmill task by dividing the submaximal exertion value by the maximal exertion value for each of $H R, \dot{V}_{E}, R E R$, and RPE and then converted each to a $z$-score. The composite physiological exertion score was then derived by averaging the $\mathrm{z}$-scores of relative effort for $\mathrm{HR}, \dot{\mathrm{V}}_{\mathrm{E}}$, and RER. 
2.3.3. Accuracy of Perceived Exertion. To quantify the degree of accuracy in perceived exertion, the composite physiological exertion $\mathrm{z}$-score was subtracted from the RPE z-score [7]. In this way, positive values would reflect overperception of exertion, and negative values reflect underperception of exertion.

Accuracy of perceived exertion $=\mathrm{RPE}_{\mathrm{z}}-$ composite physiological exertion . $_{\text {. }}$

2.4. Questionnaires. While maintained in energy balance, study participants were asked to complete questionnaires. To assess self-reported vitality and physical function, we administered the Short-form health survey (SF-36) which is a 36-item survey measuring 8 subscales (Medical Outcomes Trust, Boston, MA). Consistent with our hypotheses for this study, we focused on the physical function, mental health, and vitality subscale scores. Raw scores were converted to a percentage with high percentages to good perceived physical function, mental health, and vitality/energy.

To assess depression we used the Beck Depression Inventory-II (BDI; [13]). Each of the 21 items corresponds to a symptom of depression. Participants rated the extent to which each symptom affected them on a scale from 0 (no symptom) to 3 (severe symptom). The sum of all items provides an estimate of the presence and severity of depressive symptoms, with high scores indicative of more depression.

To assess weight control behaviors, we administered the Weight Locus of Control (WLOC) survey [14] and two dietary control surveys: the Three-Factor Eating Questionnaire-revised (TFEQ-R; [15]) and the Weight Efficacy Lifestyle (WEL; [16]) questionnaires. The WLOC measures the degree to which people attribute their weight status to internal versus external factors. High scores indicate attribution of weight control to external forces beyond their control. The 18-item TFEQ- $\mathrm{R}$ provides scores on three factors: cognitive restraint, uncontrolled eating (i.e., disinhibition), and emotional eating. Higher scores indicate greater cognitive restraint, more control over eating and less emotional eating. We elected to use this revised version rather than the original TFEQ because this version is shorter and so eased the paperwork burden on the participants. The WEL questionnaire contains 20 items that address dietary self-efficacy (i.e., confidence to resist food), yielding an overall mean and five subscale scores. Higher scores reflect greater confidence to resist food. Given that we were interested in general dietary control as a weight control behavior, we considered only the overall mean of this survey.

2.5. Statistics. Simple Pearson correlations were used to assess the degree of association between each of the questionnaire outcomes, and the RPE and accuracy of perceived exertion during the submaximal task. Multiple linear regression analyses were then used to determine whether any of the questionnaire outcomes contributed to the variance in accuracy of perceived exertion. The adjusted $R^{2}$ of the model was used to denote the percent of variance in the dependent variable explained by the independent variables.
TABLE 1: Characteristics of the study population.

\begin{tabular}{lc}
\hline Variable & Mean \pm SD \\
\hline Ethnicity & $49.2 \%$ European American \\
Age $($ years $)$ & $34.94 \pm 6.21$ \\
BMI $\left(\mathrm{kg} / \mathrm{m}^{2}\right)$ & $23.85 \pm 1.01$ \\
Walk $4.84 \mathrm{~km} / \mathrm{h} \mathrm{RPE}$ & $9.04 \pm 1.89(45.2 \%$ of max $)$ \\
Walk $4.84 \mathrm{~km} / \mathrm{h}$ composite & \\
physiological exertion & $0.01 \pm 0.79(49.4 \%$ of max $)$ \\
(Z-scores) & \\
Walk $4.84 \mathrm{~km} / \mathrm{h}$ accuracy of & $-0.06 \pm 1.18($ range: -3.1 to 2.4$)$ \\
perceived exertion $(\mathrm{Z}$-scores $)$ & $33.04 \pm 4.99$ \\
VOO 2 max & $96.71 \pm 10.08 \%$ \\
Physical function & $66.25 \pm 16.62 \%$ \\
Vitality & $82.69 \pm 12.09 \%$ \\
Mental health & $3.68 \pm 3.92$ \\
Beck depression inventory & $6.90 \pm 2.52$ \\
Weight locus of control & $19.79 \pm 3.03$ \\
Cognitive restraint & $27.92 \pm 4.37$ \\
Uncontrolled eating & $8.35 \pm 2.47$ \\
Emotional eating & $6.76 \pm 1.47$ \\
Dietary self-efficacy &
\end{tabular}

All data are reported as mean \pm standard deviation, and the alpha level was set at 0.05 for all analyses. All analyses were made with SPSS version 10.0.

\section{Results}

Characteristics of the study population are presented in Table 1. Sixty-two of the women were European American and 64 were African American. As a group, participants perceived their exertion to be approximately $45 \%$ of maximum during the $4.84 \mathrm{kph}$ treadmill task, and their physiological exertion was similar ( $49 \%$ of maximum). On average across the whole group, RPE was representative of physiological exertion, depicted by the mean of 0 for accuracy of perceived exertion. As expected in a normally distributed population, approximately $49 \%$ of participants overperceived exertion, with $15.1 \%$ of the entire sample overperceiving exertion by at least 1 standard deviation. Neither RPE nor accuracy of perceived exertion differed by whether participants had engaged in exercise during the weight loss intervention.

As shown in Table 2, simple Pearson correlations revealed that RPE was inversely associated with each of the dietary control indices, vitality, and weakly with mental health. In addition, there was a tendency for women who report an external weight locus of control to have higher RPE. Thus, women with high RPE tended to report poorer weight control behaviors, lower vitality, and poorer mental health compared to those with low RPE. These questionnaire outcomes were not associated with the composite physiological exertion score to a statistically significant degree. The accuracy of perceived exertion score was inversely associated with each of the dietary control indices, vitality, and mental health, as shown in Figures 1(a)-1(f). In other words, women 
TABLE 2: Simple correlations between RPE and questionnaire outcomes.

\begin{tabular}{lc}
\hline Variable & $r$ \\
\hline Physical function & 0.045 \\
Vitality & $-0.204^{*}$ \\
Mental health & $-0.164^{\dagger}$ \\
Beck depression inventory & -0.110 \\
Weight locus of control & $0.167^{\dagger}$ \\
Cognitive restraint & $-0.203^{*}$ \\
Uncontrolled eating & $-0.166^{\dagger}$ \\
Emotional eating & $-0.224^{*}$ \\
Dietary self-efficacy & $-0.246^{* *}$ \\
\hline
\end{tabular}

${ }^{*} P<.05 ;{ }^{* *} P<.01 ;{ }^{\dagger} .05<P<.10$

who overperceived exertion reported less dietary control, lower vitality, and poorer mental health. Ethnicity, age, and physical function were not associated with RPE or the accuracy of perceived exertion score.

Multiple linear regression models were used to determine whether the questionnaire outcomes predicted variance in the accuracy of perceived exertion. Due to the collinearity between the dietary control indices, we used only the TFEQ-cognitive restraint score in the regression models as representative of general dietary control. In addition, vitality and mental health were found to be correlated in this sample $(r=0.547, P<.001)$, and so each of these variables was included in separate models. As shown in Table 3 (model 1), low cognitive restraint and low vitality were each independently associated with the overperception of exertion, and together accounted for almost $8 \%$ of the variance in the overperception of exertion. In model 2, vitality was replaced with mental health, which subsequently was also found to be associated with the overperception of exertion, independently of cognitive restraint. Together, cognitive restraint and mental health accounted for 7\% of the variance in overperception of exertion. When ethnicity, age, and weight loss intervention group (i.e., exercise versus no exercise) were included in these models, these variables were not predictive of accuracy of perceived exertion and did not affect the significance of the associations between cognitive restraint, vitality, and mental health and accuracy of perceived exertion.

\section{Discussion}

This study examined whether self-reported physical or mental health, or weight control behaviors were related to the tendency to over- or underperceive exertion. To date, very little research has attempted to relate accuracy of perceived exertion with psychological variables. Identification of factors that may influence accuracy of perceived exertion is important however, because ultimately, the overperception of exertion may serve as a barrier against engagement in physical activity and thus a barrier against maintaining a healthy lifestyle and body weight. Indeed, our previous work has shown that women who overperceive exertion during physical activity are more likely to regain previously lost weight [7]. In the current study, we found support for our a priori hypothesis, that women who overperceived exertion reported lower vitality, poorer mental health, and poorer dietary control compared to those who underperceived exertion. Although the associations of these variables with the overperception of exertion was modest, the findings are of importance because they infer that psychological constructs may be involved in accuracy of perceived exertion, which ultimately may influence how likely a person is to engage in physical activity.

The finding that low self-reported vitality was associated with the overperception of exertion is not altogether surprising. Women who feel more energetic would be expected to perceive physical activity as easier than those who are fatigued or less energetic. A few previous studies imply the existence of such an association between vitality and perceived exertion $[17,18]$. This current study extends these findings by showing that vitality was related to perceived exertion but not to physiological measures of exertion, suggesting that there may be a psychological component captured by the vitality score that underlies perceived exertion.

It is unlikely that low vitality among those who overperceived exertion in this study was related to perceived poor functional capacity, given the lack of association between accuracy of perceived exertion and self-reported physical function during tasks of daily living. However, it is plausible that the association between accuracy of perceived exertion and vitality is a function of the amount or intensity of physical activity that people engage in during their daily lives. Amount of free-living physical activity or energy expenditure is positively associated with greater perceived energy or vitality [19-21] and inversely associated with RPE [3]. The intensity of free-living activity, in addition to the amount of free-living activity, might also be important, with less intense free-living activity being associated with higher RPE during exercise [22]. Together with the current findings, research supports a role for engagement in physical activity as mediating the relationship between vitality and accuracy in the perception of exertion. Future research is warranted, particularly to determine whether poor vitality precedes, or is consequent to, sedentary behavior. Furthermore, it will be important to consider whether it is the amount of, or the intensity of, free-living physical activity that mediates the relationship between accuracy of perceived exertion and vitality.

In a review of the psychological correlates of perceived exertion, Morgan [23] showed that anxiety, depression, and neuroticism have each been found to be associated with perceived exertion. Our study extends these findings to show that poor mental health was related specifically to the overperception of exertion, in comparison to the physiological response to the exercise challenge. The mechanism underlying the association between mental health and accuracy in the perception of exertion is not known. However, given the collinearity between mental health and vitality in this sample, it is possible that the combination of these two variables represent another underlying construct that was 


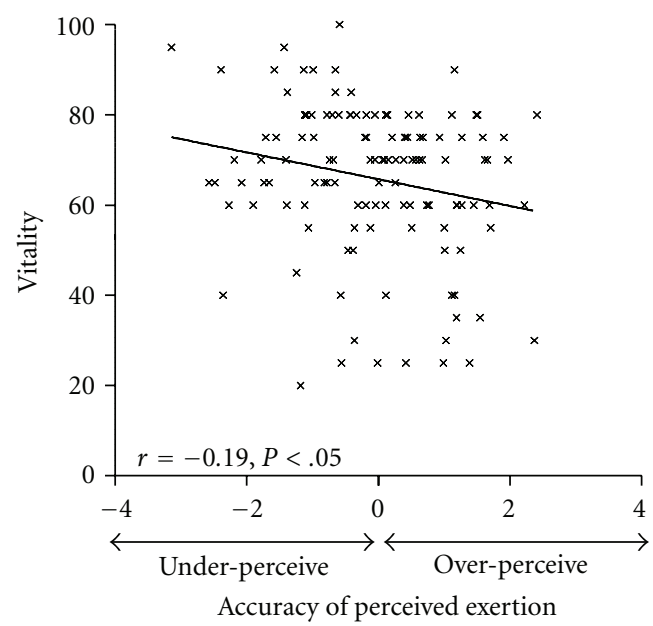

(a)

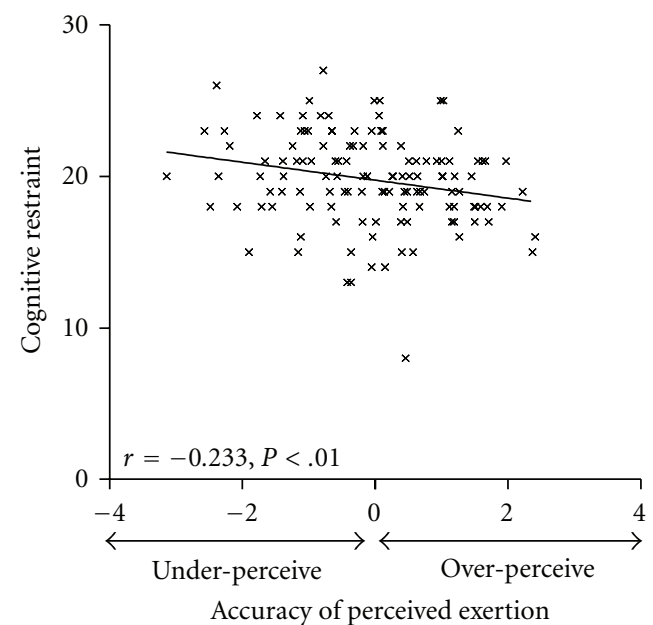

(c)

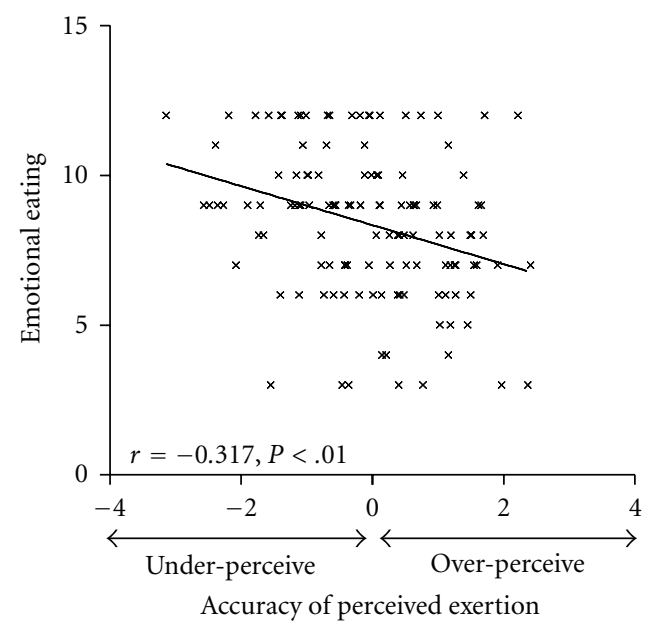

(e)

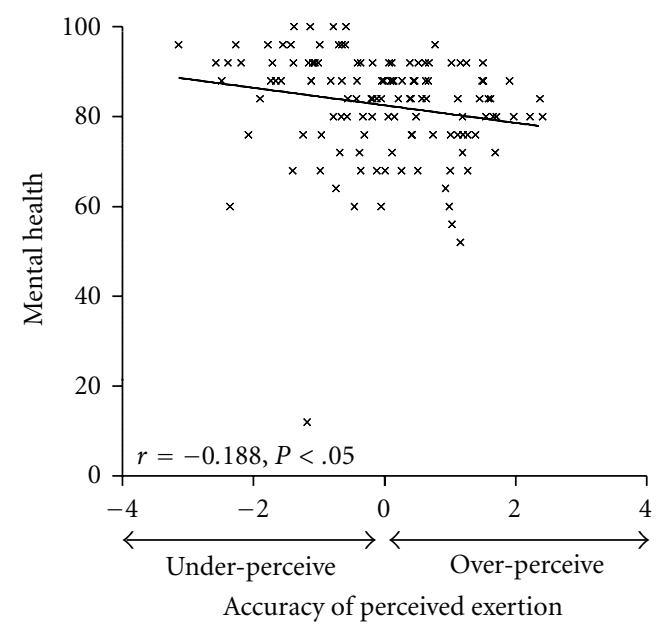

(b)

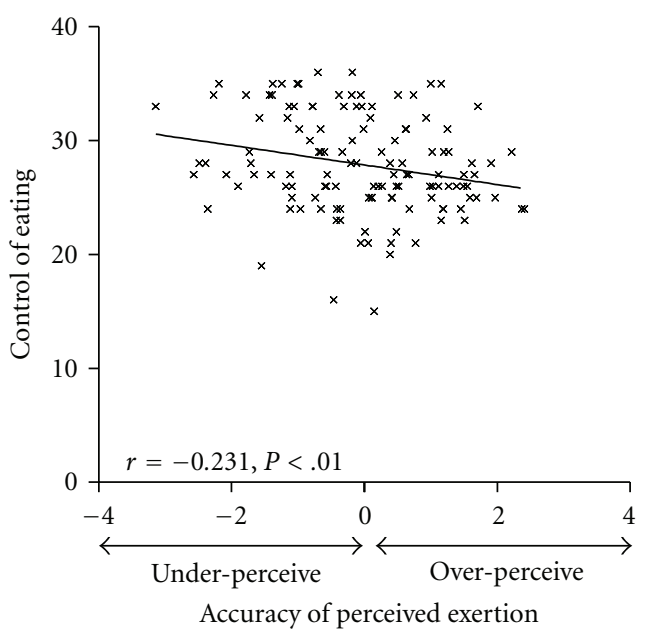

(d)

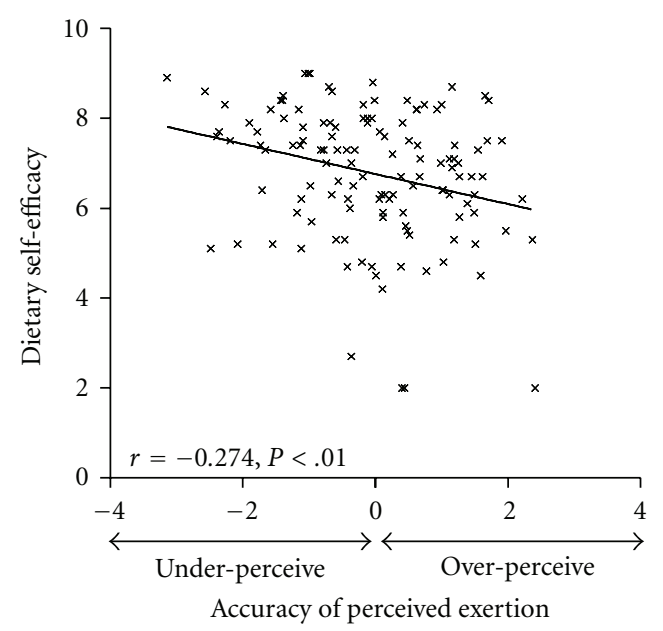

(f)

Figure 1: Simple Pearson correlations between accuracy of perceived exertion and vitality (a), mental health (b), cognitive restraint (c), control of eating (d), emotional eating (e), and dietary self-efficacy (f). The overperception of exertion was associated with lower vitality, poorer mental health, less cognitive restraint, less control of eating, more emotional eating, and lower dietary self-efficacy. 
TABLE 3: Multiple linear regression models for the dependent variable "accuracy of perceived exertion".

\begin{tabular}{lccccc}
\hline $\begin{array}{l}\text { Model } \\
\text { variables }\end{array}$ & Intercept & Slope & $\begin{array}{c}\text { Model } \\
\text { adj } \cdot R^{2}\end{array}$ & $\begin{array}{c}\text { Standardized } \\
\beta\end{array}$ & $P$ \\
\hline Model 1 & 2.683 & & 0.077 & & .003 \\
$\quad \begin{array}{l}\text { Cognitive } \\
\text { restraint } \\
\quad \text { Vitality }\end{array}$ & -0.093 & & -0.236 & .007 \\
\hline $\begin{array}{l}\text { Model 2 } \\
\text { Cognitive }\end{array}$ & 3.099 & -0.014 & & -0.194 & .027 \\
$\begin{array}{l}\text { Cestraint } \\
\quad \text { Mental }\end{array}$ & -0.087 & 0.070 & & .004 \\
\begin{tabular}{l} 
Health \\
\hline
\end{tabular} & -0.018 & & -0.223 & .011 \\
\end{tabular}

not examined here. For example, low mental health and low vitality scores may reflect a more negative personality, which was also possibly reflected by their overperception of exertion during the exercise challenge. Alternately, relatively poor mental health, like vitality, might result in less free-living physical activity, either by duration or intensity, resulting in inaccuracy in the perception of exertion. Future research should explore this finding further to examine whether mental health directly influences accuracy of perceived exertion, or whether its effect is mediated by another underlying construct, such as regulation of free-living physical activity.

The association between low dietary restraint and overperception of exertion, on the surface, appears difficult to explain. Given that all four indices of dietary restraint were shown to be related to accuracy of perceived exertion, and that each index measures a different (although related) dietary behavior, it could be the case that the lack of dietary restraint is a proxy indicator of poor weight control behaviors in general. In support of this possibility, a review of dietary disinhibition studies suggested that people with high disinhibition or uncontrolled eating are more likely to be sedentary than those with better dietary control [24]. Similarly, a study of female college students found that those with a high degree of dietary restraint also exercised more, which may indicate that women with good dietary control also use physical activity to control their weight [25]. An alternative explanation for our finding may be that women who find exercise to be particularly difficult are more critical of their own feeding behavior irrespective of whether that perception is realistic. Regardless of the underlying reason for this association, it highlights the fact that, although successful weight loss depends on lifestyle changes, including increased physical activity, perceived difficulty of exercise and lack of dietary control are both barriers to lifestyle change, and both may need to be addressed before weight loss can be maintained.

In this study a weak association was found between weight-specific locus of control and RPE. The finding is in support of that shown by Hassmén and Koivula [11], who found that women with an external locus of control had higher RPE but lower heart rates than that expected in the general population, during a cycle task. Although their results imply that a generalized external locus of control is associated with the overperception of exertion, we did not find that a weight-specific locus of control was associated with the overperception of exertion. It may be that the weight-specific locus of control measure we used in this study is too specific, particularly among a relatively homogenous formerly overweight, sedentary female population, to identify an association between weight locus of control and accuracy in perceived exertion. Despite the limited findings of the current study, future investigations of locus of control and accuracy of perceived exertion might be valuable to identify an underlying personality construct that relates to the overperception of exertion.

This study was strengthened by the fact that we objectively assessed accuracy of perceived exertion, through comparison with a composite physiological exertion score, rather than comparing RPE to heart rate. Furthermore, testing was performed while participants were in weight maintenance, with food provided by the investigators, which would have minimized any extraneous effect of recent food intake on study outcomes. The relative homogeneity of the study population (i.e., premenopausal women who underwent a recent weight loss intervention to achieve a BMI $<25.0 \mathrm{~kg} / \mathrm{m}^{2}$ ), limits the generalizability of these results to the population at large. This homogeneity may have also contributed to the modest nature of the associations shown here, and studies with a more heterogeneous population would be valuable to explore both the generalizability of these findings and strength of the associations further. Also, although we were able to identify correlates of the overperception of exertion, further research will be required to show cause and effect.

Despite these limitations, the results of this study provide an interesting thread for future research. Factors that are associated with the accuracy of perceived exertion may be valuable in terms of identifying people who are likely to experience difficulty in changing their lifestyle to defend against weight gain or weight regain. Future research is needed to explore whether RPE, or more specifically, accuracy of perceived exertion directly or indirectly influences motivation to undertake physical activity under free-living conditions.

\section{Acknowledgments}

This paper was in part supported by National Institute of Health Grant RO1 DK 51684-10, and General Clinical Research Center Grant M01 RR-00032. No financial conflicts exist.

\section{References}

[1] R. P. Troiano, D. Berrigan, K. W. Dodd, L. C. Mâsse, T. Tilert, and M. Mcdowell, "Physical activity in the United States measured by accelerometer," Medicine and Science in Sports and Exercise, vol. 40, no. 1, pp. 181-188, 2008.

[2] M. Pratt, C. A. Macera, and C. Blanton, "Levels of physical activity and inactivity in children and adults in the United 
States: current evidence and research issues," Medicine and Science in Sports and Exercise, vol. 31, supplement 1, no. 11, pp. S526-S533, 1999.

[3] G. R. Hunter, R. L. Weinsier, P. A. Zuckerman, and B. E. Darnell, "Aerobic fitness, physiologic difficulty and physical activity in Black and White women," International Journal of Obesity Related to Metabolic Disorders, vol. 28, no. 9, pp. 11111117, 2004.

[4] G. Borg and H. Linderhorm, "Perceived exertion and pulse rate during graded exercise in various age groups," Acta Medica Scandinavica. Supplement, vol. 472, pp. 194-206, 1967.

[5] R. J. Robertson and B. J. Noble, "Perception of physical exertion: methods, mediators, and applications," Exercise and Sport Sciences Reviews, vol. 25, pp. 407-452, 1997.

[6] J. S. Skinner, R. Hutsler, V. Bergsteinova, and E. R. Buskirk, "The validity and reliability of a rating scale of perceived exertion," Medicine and Science in Sports, vol. 5, no. 2, pp. 94 96, 1973.

[7] D. W. Brock, P. C. Chandler-Laney, J. A. Alvarez, B. A. Gower, G. A. Gaesser, and G. R. Hunter, "Perception of Exercise Difficulty Predicts Weight Regain in Formerly Overweight Women," Obesity, vol. 18, pp. 982-986, 2009.

[8] C. S. Carver, A. E. Coleman, and D. C. Glass, "The coronaryprone behavior pattern and the suppression of fatigue on a treadmill test," Journal of Personality and Social Psychology, vol. 33, no. 4, pp. 460-466, 1976.

[9] R. K. Dishman, R. E. Graham, J. Buckworth, and J. WhiteWelkley, "Perceived exertion during incremental cycling is not influenced by the type A behavior pattern," International Journal of Sports Medicine, vol. 22, no. 3, pp. 209-214, 2001.

[10] R. K. Dishman, R. E. Graham, R. G. Holly, and J. G. Tieman, "Estimates of Type A behavior do not predict perceived exertion during graded exercise," Medicine and Science in Sports and Exercise, vol. 23, no. 11, pp. 1276-1282, 1991.

[11] P. Hassmén and N. Koivula, "Ratings of perceived exertion by women with internal or external locus of control," Journal of General Psychology, vol. 123, no. 4, pp. 297-307, 1996.

[12] R. L. Weinsier, G. R. Hunter, P. A. Zuckerman et al., "Energy expenditure and free-living physical activity in black and white women: comparison before and after weight loss," American Journal of Clinical Nutrition, vol. 71, no. 5, pp. 1138-1146, 2000.

[13] A. T. Beck, R. A. Steer, and G. K. Brown, BDI-II Manual, The Psychological Corporation, London, UK, 1996.

[14] E. B. Saltzer, "The weight locus of control (WLOC) scale: a specific measure for obesity research," Journal of Personality Assessment, vol. 46, no. 6, pp. 620-628, 1982.

[15] J. Karlsson, L. -O. Persson, L. Sjöström, and M. Sullivan, "Psychometric properties and factor structure of the ThreeFactor Eating Questionnaire (TFEQ) in obese men and women. Results from the Swedish Obese Subjects (SOS) study," International Journal of Obesity Related to Metabolic Disorders, vol. 24, no. 12, pp. 1715-1725, 2000.

[16] M. M. Clark, D. B. Abrams, R. S. Niaura, C. A. Eaton, and J. S. Rossi, "Self-efficacy in weight management," Journal of Consulting and Clinical Psychology, vol. 59, no. 5, pp. 739-744, 1991.

[17] F. Hegbom, K. Stavem, S. Sire, M. Heldal, O. M. Orning, and K. Gjesdal, "Effects of short-term exercise training on symptoms and quality of life in patients with chronic atrial fibrillation," International Journal of Cardiology, vol. 116, no. 1, pp. 86-92, 2007.

[18] R. Eston, J. Faulkner, A. St Clair Gibson, T. Noakes, and G. Parfitt, "The effect of antecedent fatiguing activity on the relationship between perceived exertion and physiological activity during a constant load exercise task," Psychophysiology, vol. 44, no. 5, pp. 779-786, 2007.

[19] P. J. O'Connor and T. W. Puetz, "Chronic physical activity and feelings of energy and fatigue," Medicine and Science in Sports and Exercise, vol. 37, no. 2, pp. 299-305, 2005.

[20] N. Kerse, C. R. Elley, E. Robinson, and B. Arroll, "Is physical activity counseling effective for older people? A cluster randomized, controlled trial in primary care," Journal of the American Geriatrics Society, vol. 53, no. 11, pp. 1951-1956, 2005.

[21] K. B. Schmaling, J. I. Fiedelak, J. Bader, and D. Buchwald, "A longitudinal study of physical activity and body mass index among persons with unexplained chronic fatigue," Journal of Psychosomatic Research, vol. 58, no. 4, pp. 375-381, 2005.

[22] D. E. Stephens, K. F. Janz, and L. T. Mahoney, "Goal orientation and ratings of perceived exertion in graded exercise testing of adolescents," Perceptual and Motor Skills, vol. 90, no. 3, part 1, pp. 813-822, 2000.

[23] W. P. Morgan, "Psychological components of effort sense," Medicine and Science in Sports and Exercise, vol. 26, no. 9, pp. 1071-1077, 1994.

[24] E. J. Bryant, N. A. King, and J. E. Blundell, "Disinhibition: its effects on appetite and weight regulation," Obesity Reviews, vol. 9, no. 5, pp. 409-419, 2008.

[25] J. A. McLean and S. I. Barr, "Cognitive dietary restraint is associated with eating behaviors, lifestyle practices, personality characteristics and menstrual irregularity in college women," Appetite, vol. 40, no. 2, pp. 185-192, 2003. 


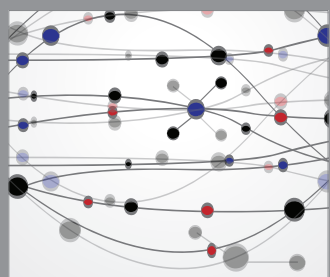

The Scientific World Journal
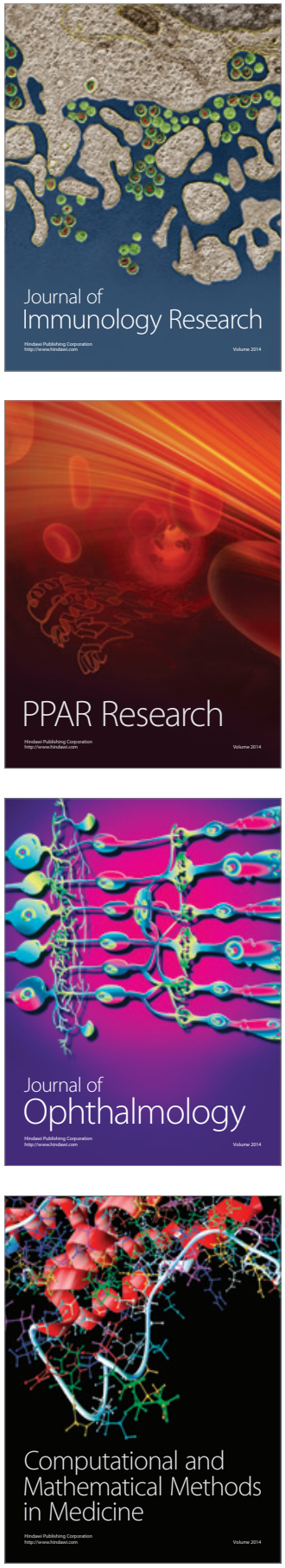

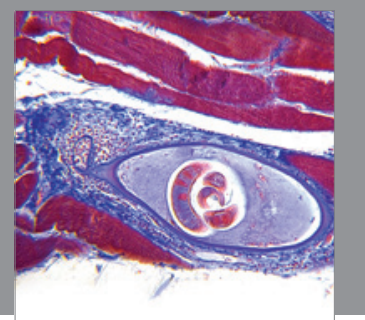

Gastroenterology

Research and Practice
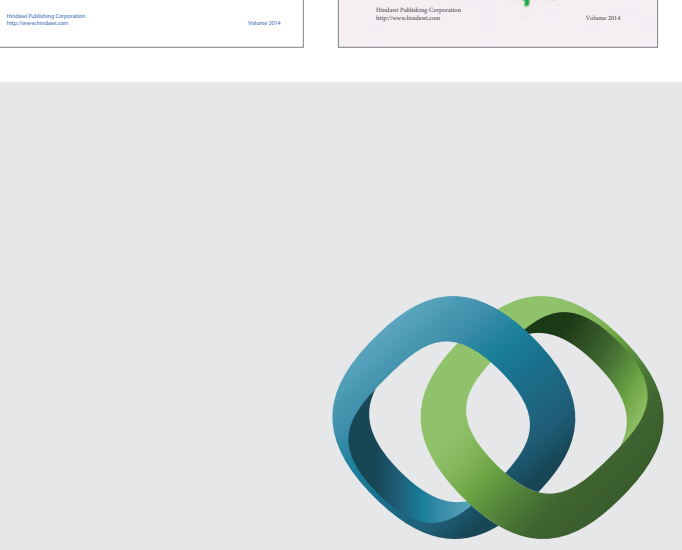

\section{Hindawi}

Submit your manuscripts at

http://www.hindawi.com
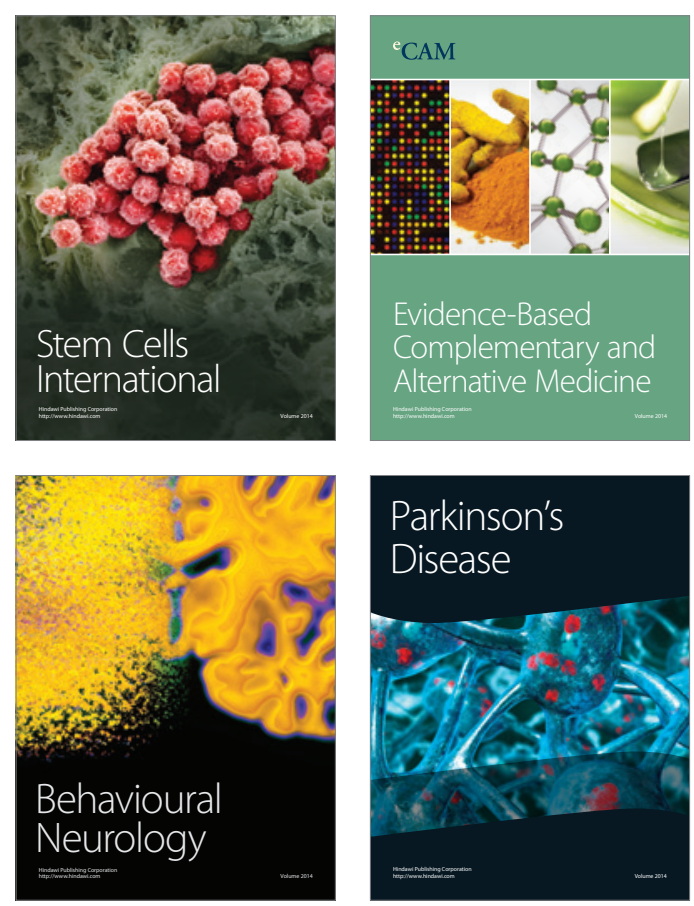

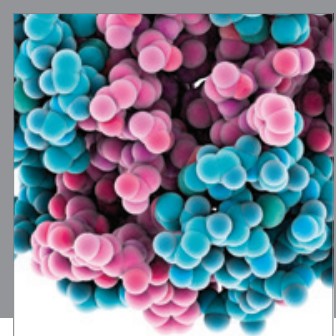

Journal of
Diabetes Research

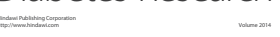

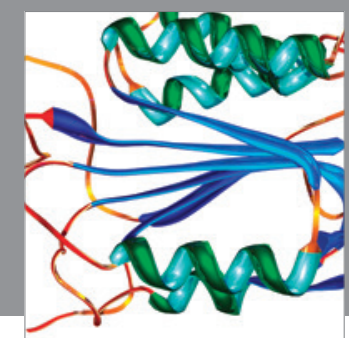

Disease Markers
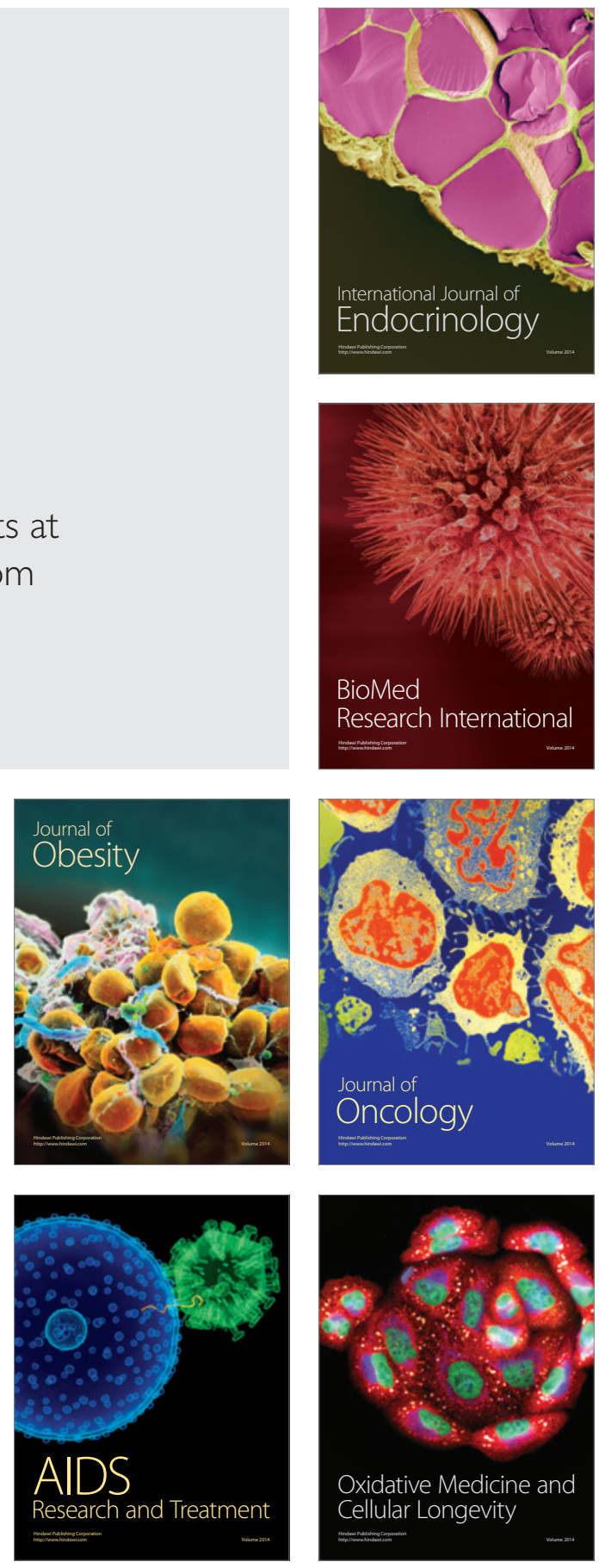